\title{
The time required for perceptual (nonmotoric) processing in IOR
}

\author{
Thomas M. Spalek and Vincent Di Lollo \\ Simon Fraser University, Burnaby, British Columbia, Canada
}

\begin{abstract}
In an inhibition of return (IOR) paradigm, we used a threshold-tracking procedure combined with backward masking to measure the speed of perceptual processing in IOR independent of motoric factors. Instead of the conventional reaction time measure, this procedure yielded the critical exposure duration $\left(\mathrm{DUR}_{\mathrm{c}}\right)$ that is required in order for a target to be identified reliably before the onset of a trailing mask. In Experiment 1, the facilitation effects conventionally found at short cue-target onset asynchrony (CTOA) were evidenced by shorter values of DUR $\mathrm{c}_{\mathrm{c}}$ at cued relative to uncued locations. Conversely, the retardation effects conventionally found at long CTOA were evidenced by correspondingly longer values of DUR . In Experiment 2, the $D_{U} R_{c}$ results strongly suggest that the directional reading bias previously observed in IOR studies is due, at least in part, to perceptual rather than motoric factors.
\end{abstract}

Visual search is something we do routinely every day. We select a coin for the parking meter from a handful of coins, or we single out an icon from among a crowd of other icons on our computer desktop. Laboratory studies have been aimed at discovering the rules that govern the efficiency of visual search. One such rule, known as inhibition of return (IOR; Posner \& Cohen, 1984), is held to be effective in preventing observers from reexamining locations that have already been searched. A rule such as this would promote the examination of novel locations, thereby improving search efficiency.

In a typical IOR experiment, the initial display consists of a central fixation point and two placeholders, one on either side of fixation. A cue stimulus appears briefly in one of the peripheral locations and then reappears briefly at fixation. A target stimulus is then presented, and the observer is required to respond to it as quickly as possible. IOR describes the finding that the observer's reaction time (RT) to a target presented at the previously cued location is slower than it is to a target presented at an uncued location (Posner \& Cohen, 1984).

In the predominant view, this increased latency is attributed to some form of inhibitory mechanism acting at either the perceptual (see, e.g., Abrams \& Dobkin, 1994) or the motor (see, e.g., Taylor \& Klein, 1998) level. However, a determination of whether the locus of IOR is perceptual or motor (or both) has been hampered by the common practice of using RT as the dependent measure. Because motor factors are inherently involved in RT, an uncontaminated estimate of the involvement of perceptual factors has been difficult to obtain (see, e.g., Müller \& von Mühlenen, 1996).

The perceptual and motor components of IOR were decoupled in a study by Handy, Jha, and Mangun (1999), who used identification of a masked target, rather than RT, as the primary response measure. By employing signaldetection methodology, a measure of perceptual sensitivity $\left(d^{\prime}\right)$ was obtained that was independent of motor factors. Handy et al.'s results revealed both classes of effects typically found in exogenous cuing studies: facilitation (larger $d^{\prime}$ at the cued relative to the uncued location) at a short cue-target onset asynchrony (CTOA) and IOR (relatively smaller $d^{\prime}$ at the cued location) at a long CTOA. On the basis of these results, Handy et al. concluded that the mechanism underlying IOR affects the perceptual quality of a target stimulus independently of motoric factors.

Although the signal-detection methodology ( $d^{\prime}$ measure) employed by Handy et al. (1999) did provide evidence for a perceptual component of IOR and for reduced sensitivity at the cued location, it did not provide evidence regarding the period of time required for the perceptual processing of the target at the cued and uncued locations. The present need is for estimates of the speed of the perceptual system at the cued relative to the uncued target locations in the absence of motoric factors. If it is the case that IOR reflects a modulation of perceptual sensitivity at the cued location relative to the uncued location, it is reasonable to expect that the speed of perceptual processing should be modulated correspondingly.

In the present work, we adopted a novel approach to the study of IOR, which yielded temporal estimates of perceptual processing while remaining unaffected by motor considerations. To wit, rather than using a signal-detection procedure, we used a dynamic threshold-tracking procedure (PEST; Taylor \& Creelman, 1967) that converged on the critical exposure duration $\left(D_{U R}\right)$, which yielded $80 \%$ correct identification of a masked target. As such, the

T. M. Spalek, tspalek@sfu.ca 
DUR $_{c}$ values obtained in the present work provide the first direct estimate of the speed of perceptual processing in IOR. Because DUR does not involve RT, it is independent of the planning and execution of motor responses.

\section{EXPERIMENT 1}

In Experiment 1, we employed the cuing paradigm described above to assess both facilitation and retardation (IOR) effects in target identification. We reasoned that if the speed of perceptual processing is modulated by cuing, DUR $_{c}$ should be shorter at the cued than at the uncued location when CTOA is short (facilitation effect). Conversely, DUR $\mathrm{C}_{\mathrm{c}}$ should be longer at the cued than at the uncued location when CTOA is long (retardation effect).

\section{Method}

Observers. Sixty undergraduate volunteers participated for class credit or payment. All reported normal or corrected-to-normal vision. Thirty-two observers were randomly assigned to the long CTOA group $(\mathrm{CTOA}=950 \mathrm{msec})$, and 28 were assigned to the short CTOA group $(\mathrm{CTOA}=100 \mathrm{msec})$.

Apparatus and Stimuli. All stimuli were displayed on a computer monitor at a screen refresh rate of $60 \mathrm{~Hz}$. The display sequence and timing are illustrated in Figure 1. The placeholders were white outlines of circles approximately $1.8^{\circ}$ in diameter; they were located approximately $5.7^{\circ}$ to either side of a central fixation cross measuring $0.5^{\circ} \times 0.5^{\circ}$. The cues were solid white disks of approximately $1^{\circ}$ diameter. The target was either a solid white square or a solid white diamond with each side measuring approximately $1^{\circ}$. The masks consisted of the two targets superimposed on one another. All stimuli were presented on a black background (approximately $0.5 \mathrm{~cd} / \mathrm{m}^{2}$ ) at a luminance of $50 \mathrm{~cd} / \mathrm{m}^{2}$ as measured by a Minolta LS 100 luminance meter. Observers sat in a darkened room and viewed the displays from a distance of approximately $60 \mathrm{~cm}$.

Procedure. Each trial began with a display of the fixation cross and the placeholders and continued with a brief presentation of the cue within either the left or the right placeholder. In the long (but not in the short) CTOA group, the cue was re-presented briefly at fixation (central reorienting). The display sequence continued for both groups with a brief presentation of the target (either a square or a diamond) inside one of the two peripheral placeholders, which was followed immediately by the mask, presented inside both placeholders. Within an experimental session, the cue was presented an equal number of times on the left and on the right.

Observers indicated whether the target was a square or a diamond by pressing the corresponding key on the keyboard. A dynamic threshold-tracking procedure (PEST) varied the exposure duration of the target in response to the participant's performance; the procedure was carried out separately for the cued and uncued trials, which were intermixed within the experimental session. At the beginning of a PEST run, the exposure duration was set at $224 \mathrm{msec}$ and was changed dynamically according to the rules of PEST (Taylor \& Creelman, 1967). PEST increased the exposure duration of the target if accuracy was below $80 \%$ and decreased exposure duration if accuracy was above $80 \%$. The initial step for changing the target's exposure duration was set at $64 \mathrm{msec}$. At the end of a run, PEST yielded the critical exposure duration $\left(D_{U} R_{c}\right.$ ) at which the target was identified correctly on $80 \%$ of the trials; again, this was done separately for the cued and uncued conditions. Three estimates of DUR $_{c}$ were obtained for each observer in each condition.

\section{Results and Discussion}

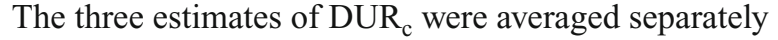
for the cued and uncued conditions for each observer. We then removed the data for observers who deviated by more than two standard deviations from the mean of their respective group in the difference between the cued and uncued conditions. As a result, two observers were dropped from the long CTOA group, leaving 30 observers in the long and 28 in the short CTOA groups. The values of $\mathrm{DUR}_{\mathrm{c}}$ for each condition, averaged over observers, are illustrated in Figure 2.

The results were submitted to a 2 (CTOA: long or short) $\times 2$ (cuing: cued or uncued) ANOVA. The analysis revealed a significant effect of cuing $[F(1,56)=14.18$, $\left.M S_{\mathrm{e}}=426.94, p<.001\right]$ and a significant CTOA $\times$ cuing interaction effect $\left[F(1,56)=85.36, M S_{\mathrm{e}}=426.94, p<\right.$ $.001]$. The interaction was due to the characteristic biphasic pattern common in IOR studies. That is, in the short CTOA group, $\mathrm{DUR}_{\mathrm{c}}$ was shorter for targets presented in the cued than in the uncued locations (facilitation effect) $[t(27)=$ 8.03, $p<.001$ ], whereas the reverse was true for the long CTOA group (retardation effect) $[t(29)=4.54, p<.001]$.

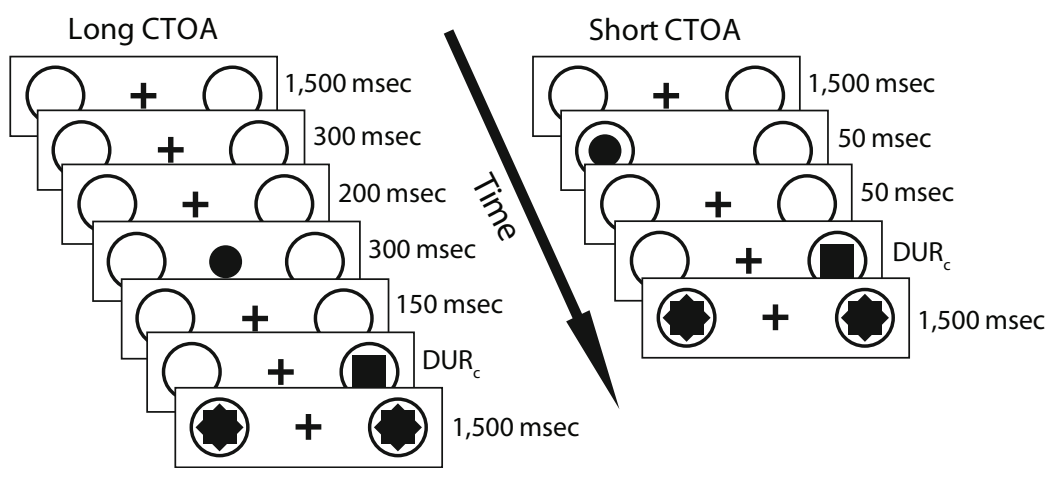

Figure 1. Schematic diagram of the display sequence in Experiment 1. Here and in Figures 2 and 3, CTOA = cue-target onset asynchrony; $D_{U R}=$ critical exposure duration required for identifying the target to a criterion of $80 \%$ before the onset of the trailing masks. 


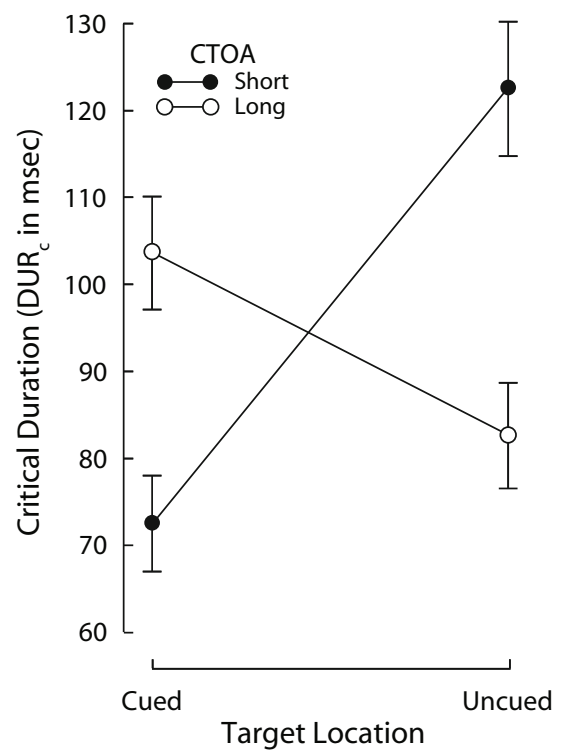

Figure 2. Results of Experiment 1. Error bars represent one standard error of the mean.

Earlier findings have linked IOR to the sensitivity of the system and hence to the perceptual quality of visual processing (Handy et al., 1999). The present work builds on these earlier findings by providing direct estimates of the changes in processing time that accompany changes in sensitivity: Higher sensitivity is linked with shorter values of DUR $\mathrm{c}_{\mathrm{c}}$, and lower sensitivity with longer values of DUR . . As was the case for the sensitivity $\left(d^{\prime}\right)$ measure employed in Handy et al.'s study, the present DUR $\mathrm{C}_{\mathrm{c}}$ measure is unlikely to be affected by motoric factors. One possible source of motoric influence - eye movements - can be safely discounted because of timing considerations. In the short CTOA condition, the SOA between cue and target was only $100 \mathrm{msec}$, which is too short a time to allow for an eye movement (see, e.g., Tam \& Ono, 1994). Even when DUR ${ }_{c}$ is added to the SOA, the interval falls considerably below $200 \mathrm{msec}$ in the cued conditions. In addition, the average $\mathrm{DUR}_{\mathrm{c}}$ difference between cued and uncued conditions - whether CTOA was long or short-never exceeded about $50 \mathrm{msec}$, an interval that is far too short for a corrective eye movement to a target presented in the uncued location.

The outcomes illustrated in Figure 2 establish a clear parallel between the pattern of results obtained using DUR $_{c}$ as the dependent measure and the conventional pattern of results reported in the IOR literature with RT as the dependent measure (see, e.g., Posner \& Cohen, 1984). We next asked whether such a parallel would also hold for more specific results, such as are obtained when IOR is examined in relation to directional reading bias. Spalek and Hammad (2005) have shown that the magnitude of IOR using RT as the dependent measure is greater when the attentional trajectory established by the sequence of peripheral and central cues (Figure 1, left panel) is consistent with the directional reading bias in the observer's culture. Thus, for English-reading observers, the left-toright sequence illustrated in the left panel of Figure 1 yielded a more pronounced IOR, as indexed by RT measures, than the converse right-to-left sequence. The opposite was true for Arabic-reading observers whose directional reading bias predisposed them to a right-to-left sequence. If the retardation effect observed in Experiment 1 reflects the same IOR phenomenon, then a similar directional bias should be observed using DUR $\mathrm{C}_{\mathrm{c}}$ as the dependent measure. This contingency was examined in Experiment 2.

\section{EXPERIMENT 2}

\section{Method}

Observers. Forty-two undergraduate, English-reading volunteers participated for class credit or payment. All reported normal or corrected-to-normal vision.

Apparatus and Stimuli. Same as those used in Experiment 1.

Procedure. In Experiment 2, we obtained three quadruplets of

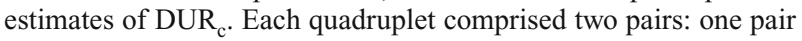
for the cued and uncued locations when the initial peripheral cue was within the left placeholder, and the other pair for when the initial cue was within the right placeholder. The CTOA was always $950 \mathrm{msec}$, corresponding to the long CTOA in Experiment 1. All other details matched those of Experiment 1.

\section{Results and Discussion}

The three estimates of each of the four DUR $\mathrm{s}$ were averaged separately for each observer. We then removed the data for any observer who deviated by more than two standard deviations from the mean of their respective group in the difference between the cued and uncued conditions when the left or the right location was cued.Three observers were consequently dropped from the analysis. The val-

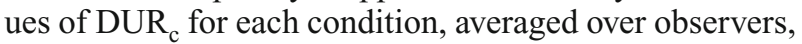
are illustrated in Figure 3.

The results were submitted to a 2 (cue location: left or right) $\times 2$ (cuing: cued or uncued) ANOVA. The analysis revealed significant effects of cue location $[F(1,38)=$ $\left.5.01, M S_{\mathrm{e}}=246.60, p=.031\right]$ and cuing $[F(1,38)=$ $\left.8.18, M S_{\mathrm{e}}=344.50, p=.007\right]$, and a significant cue location $\times$ cuing interaction effect $\left[F(1,38)=8.89, M S_{\mathrm{e}}=\right.$ $234.05, p=.005]$. The interaction was due to the retardation effect's being in evidence when the initial cue appeared at the left location [t(38) $=4.25, p<.001]$, but not when it appeared in the right location $[t(38)=0.30$, $p=.77]$.

The results of Experiment 2 can be explained on the basis of the interaction between two factors: reading bias and attentional momentum. The attentional momentum hypothesis holds that the focus of attention is repositioned more readily in the direction of an established trajectory than in the opposite direction (Posner \& Cohen, 1984; Pratt, Spalek, \& Bradshaw, 1999; Spalek \& Hammad, 2004). In Experiment 2, presentation of the initial cue over the left placeholder was followed by the central reorienting event, thus establishing a left-to-right attentional trajectory. In this case, reading bias and attentional momentum worked in conjunction, leading our English-reading observers to expect the next stimulus (the target) to occur on the right. In turn, this led to retardation (longer $\mathrm{DUR}_{\mathrm{c}} \mathrm{s}$ ) on trials in which the target occurred on the left relative to trials 


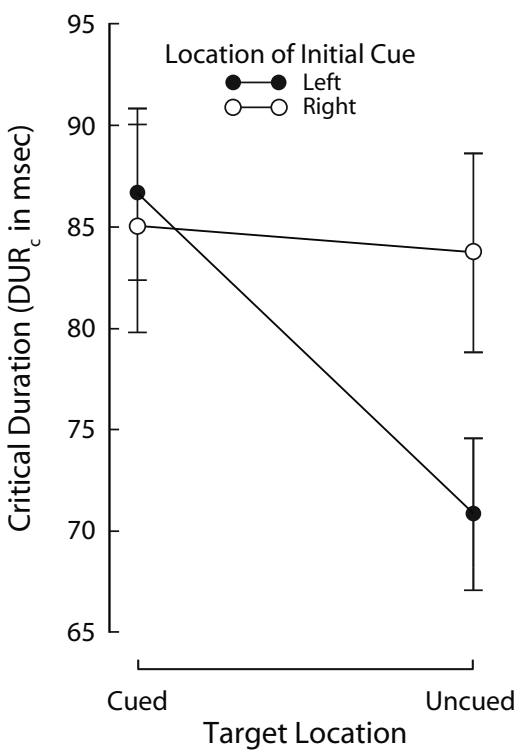

Figure 3. Results of Experiment 2. Error bars represent one standard error of the mean.

in which the target occurred on the right (Figure 3, filled symbols).

In contrast, presentation of the initial cue over the right placeholder, followed by the central reorienting event, established a right-to-left attentional trajectory. In this case, reading bias and attentional momentum worked in opposition to one another: Reading bias led observers to expect the target on the right, whereas attentional momentum led them to expect the target on the left. The two factors effectively counteracted each other, leading to a null difference between cued and uncued locations (Figure 3, open symbols). This pattern of results mirrors that reported by Spalek and Hammad (2005), buttressing the parallel between RT and DUR $\mathrm{R}_{\mathrm{c}}$ as valid measures of IOR effects. In addition, the present $D_{U R}$ results strongly suggest that the directional reading bias influence on IOR reported by Spalek and Hammad was due, at least in part, to perceptual rather than from motoric factors.

Two aspects of the results of Experiment 2 need elaboration. First, as seen in the two points on the left side in Figure 3, the DUR value for the condition in which both the initial cue and the target were displayed on the left was only slightly higher than for the condition in which both were displayed on the right. According to the reading bias/ attentional momentum account, the former $\mathrm{DUR}_{\mathrm{c}}$ (both biases against) should have been significantly higher than the latter (momentum bias against, reading bias for). Al-

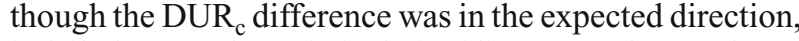
it failed to reach statistical significance and was, therefore, slightly at odds with predictions. It should be noted, however, that the direction of the difference obtained in the present study with a DUR a corresponding difference obtained with an RT measure (Spalek \& Hammad, 2005).

The second aspect in need of elaboration concerns a comparison between the results of Experiment 2 and the corresponding results in the long CTOA condition in Experiment 1 . When averaged across the location of initial cue (i.e., collapsing across the two functions in Figure 3), the retardation effect in Experiment 2 (cued $\mathrm{DUR}_{\mathrm{c}}-$ uncued $\mathrm{DUR}_{\mathrm{c}}=8.5 \mathrm{msec}$ ) was considerably less than the corresponding retardation effect in Experiment 1 (cued $\mathrm{DUR}_{\mathrm{c}}$ - uncued DUR $\mathrm{c}=21.0 \mathrm{msec}$ ). The two experiments differed only in what may be regarded as a minor detail: Each observer yielded a total of 12 estimates of DUR $_{\mathrm{c}}$ (three quadruplets) in Experiment 2 but only 6 estimates (three pairs) in Experiment 1. Thus, observers received more extended practice at the task in Experiment 2 than in Experiment 1. It is possible that the extended practice in Experiment 2 may have led to an attenuation of the retardation effect. This account, however, is speculative and in need of empirical evaluation.

\section{GENERAL DISCUSSION}

A novel approach to the study of IOR was employed in the present work, which has yielded the first direct temporal estimate of the perceptual processes involved in IOR independent of motoric processes. An earlier study that pointed to the independence of IOR from motoric influences (Handy et al., 1999) has been questioned on the grounds that motoric components may not have been entirely excluded from that study because it employed a unilateral backward mask (Ivanoff \& Klein, 2001). That concern has been addressed in the present work through the use of bilateral masks that yielded findings homologous to those of Handy et al. In this respect, the present study supplements Handy et al.'s findings by adding a temporal estimate of IOR, which in turn buttresses the conclusion that IOR can affect the perceptual quality of visual processing independent of motoric considerations. That IOR can occur without motoric involvement is further supported by Prime and Ward's (2004) finding that no effect of IOR is found on motor processes as indexed by the lateralized readiness potential of the brain's electrical response.

It needs to be stressed that the temporal estimates obtained in the present work do not differentiate between two alternatives - the time required to reposition the attentional spotlight as distinct from changes in the rate of information processing at the cued location. For example, the lower values of DUR $\mathrm{c}_{\mathrm{c}}$ obtained at short CTOAs may represent either faster deployment of attention to the cued location and/or an increased rate of processing at that location. A decision between these alternatives cannot be reached on the basis of either Handy et al.'s (1999) $d^{\prime}$ measure or the present DUR $\mathrm{c}_{\mathrm{c}}$ measure. Indeed, strictly behavioral measures such as these may in principle be unsuited for this purpose. More direct measures of brain activity, such as event-related potentials, show greater promise of being capable of separating the two alternatives (see, e.g., McDonald, Hickey, Green, \& Whitman, 2005).

\section{AUTHOR NOTE}

This work was sponsored by the President Research Grant and the Endowed Research Fellowship from Simon Fraser University to T.M.S. and a grant from the Natural Sciences and Engineering Research Council 
of Canada to V.D.L. We acknowledge the expert assistance of Shannon Gaudry in the collection of the data. Correspondence concerning this article should be addressed to T. M. Spalek, Department of Psychology, Simon Fraser University, 8888 University Drive, Burnaby, BC, V5A 1S6 Canada (e-mail: tspalek@sfu.ca).

\section{REFERENCES}

Abrams, R. A., \& Dobkin, R. S. (1994). Inhibition of return: Effects of attentional cuing on eye movement latencies. Journal of Experimental Psychology: Human Perception \& Performance, 20, 467-477.

Handy, T. C., Jha, A. P., \& Mangun, G. R. (1999). Promoting novelty in vision: Inhibition of return modulates perceptual-level processing. Psychological Science, 10, 157-161.

IVANOFF, J., \& KLEIN, R. M. (2001). The presence of a nonresponding effector increases inhibition of return. Psychonomic Bulletin \& Review, 8, 307-314.

McDonald, J. J., Hickey, C., Green, J. J., \& Whitman, J. C. (2005, November). Suppression of attention at previously attended locations: Evidence from human electrophysiology. Paper presented at the annual meeting of the Psychonomic Society, Toronto, ON, Canada.

Müller, H. J., \& VON MüHLEnEN, A. (1996). Attentional tracking and inhibition of return in dynamic displays. Perception \& Psychophysics, 58, 224-249.

Posner, M. I., \& Cohen, Y. A. (1984). Components of visual orient- ing. In H. Bouma \& D. G. Bouwhuis (Eds.), Attention and performance $X$ : Control of language processes (pp. 531-556). Hillsdale, NJ: Erlbaum.

Pratt, J., SpaleK, T. M., \& Bradshaw, F. (1999). The time to detect targets at inhibited and noninhibited locations: Preliminary evidence for attentional momentum. Journal of Experimental Psychology: Human Perception \& Performance, 25, 730-746.

Prime, D. J., \& WARD, L. M. (2004). Inhibition of return from stimulus to response. Psychological Science, 15, 272-276.

SpaleK, T. M., \& Hammad, S. (2004). Supporting the attentional momentum view of IOR: Is attention biased to go right? Perception \& Psychophysics, 66, 219-233.

SpaleK, T. M., \& Hammad, S. (2005). The left-to-right bias in inhibition of return is due to the direction of reading. Psychological Science, 16, 15-18.

TAm, W. J., \& Ono, H. (1994). Fixation disengagement and eyemovement latency. Perception \& Psychophysics, 56, 251-260.

Taylor, M. M., \& Creelman, C. D. (1967). PEST: Efficient estimates on probability functions. Journal of the Acoustical Society of America, 41, 782-787.

TAYlOR, T. L., \& KLEIN, R. M. (1998). On the causes and effects of inhibition of return. Psychonomic Bulletin \& Review, 5, 625-643.

(Manuscript received February 15, 2006; revision accepted for publication March 13, 2006. ) 